Tohoku Math. J.

67 (2015), 281-295

\title{
ON THE STRUCTURE OF LINEARIZATION OF THE SCALAR CURVATURE
}

\author{
Gabjin Yun, JeOngwook Chang And Seungsu Hwang
}

(Received September 17, 2013, revised April 14, 2014)

\begin{abstract}
For a compact $n$-dimensional manifold a critical point metric of the total scalar curvature functional satisfies the critical point equation (1) below, if the functional is restricted to the space of constant scalar curvature metrics of unit volume. The right-hand side in this equation is nothing but the adjoint operator of the linearization of the total scalar curvature acting on functions. The structure of the kernel space of the adjoint operator plays an important role in the geometry of the underlying manifold.

In this paper, we study some geometric structure of a given manifold when the kernel space of the adjoint operator is nontrivial. As an application, we show that if there are two distinct solutions satisfying the critical point equation mentioned above, then the metric should be Einstein. This generalizes a main result in [6] to arbitrary dimension.
\end{abstract}

1. Introduction. Hilbert proved that on a given compact $n$-dimensional manifold $M$ Einstein metrics are the critical points of the total scalar curvature functional, namely

$$
g \rightarrow \int_{M} s_{g} d v_{g}
$$

Here, $s_{g}$ is the scalar curvature, $d v_{g}$ is the volume form determined by the metric and orientation, and the functional is restricted to metrics whose volume is fixed, say equal to 1 .

In the 1970s, N. Koiso found the following refinement by taking the smaller domain of this functional ([7]). He introduces the space $\mathcal{C}$ of metrics with constant scalar curvature and total volume 1 . Then the restriction of the total scalar curvature functional to $\mathcal{C}$ is just the function $g \mapsto s_{g}$. With this restriction, at least formally, $g$ is a critical point if there exists a function $f$ such that

$$
z_{g}=s_{g}^{*}(f),
$$

which is called the critical point equation (CPE in short). Here $z_{g}$ is the traceless Ricci tensor of $g$, and $s_{g}^{\prime *}$ is given by

$$
s_{g}^{\prime *}(f)=D_{g} d f-\left(\Delta_{g} f\right) g-f r_{g},
$$

2010 Mathematics Subject Classification. Primary 53C25; Secondary 58E11.

Key words and phrases. Total scalar curvature functional, critical point metric, Einstein metric.

The first author was supported by the Basic Science Research Program through the National Research Foundation of Korea(NRF) funded by the Ministry of Education, Science and Technology (2011-0007465), the second author by the Ministry of Education, Science and Technology (2010-0011310), and the third and corresponding author by the Ministry of Education, Science and Technology (2010-0010792). 
where $D_{g} d$ and $\Delta_{g}$ denote the Hessian and the (negative) Laplacian, respectively, and $r_{g}$ is the Ricci curvature of $g$. We remark that by taking the trace of (2), we have

$$
\Delta_{g} f=-\frac{s_{g}}{n-1} f \text {. }
$$

Thus, if $s_{g}$ is negative, then the eigenfunction $f$ should be trivial. The linear operator $s_{g}^{\prime *}$ is, in fact, the $L^{2}$ adjoint of the linearization $s_{g}^{\prime}$ of the scalar curvature $s_{g}$ given by

$$
s_{g}^{\prime}(h)=-\Delta_{g} t r h+\delta_{g} \delta_{g} h-g\left(h, r_{g}\right)
$$

(c.f. $[1,1.174$, p. 63]), where $h$ is any symmetric bilinear form on $M$ and $\delta$ is the divergence operator.

When $g$ is Einstein, (1) is equivalent to Obata's equation and so such a solution is isometric to a standard $n$-sphere ([10]). It can be conjectured that this is the only possible case ([1]). Since $z_{g}=0$ if and only if $g$ is Einstein, (1) is related to the kernel space, $\operatorname{ker} s_{g}^{\prime *}$, of $s_{g}^{*}$. There are some results on the $\mathrm{CPE}$ with $\operatorname{ker} s_{g}^{\prime *} \neq 0$. One of basic fact due to J. P. Bourguignon ([2]) is that if $\operatorname{ker} s_{g}^{\prime *} \neq 0$, then either $(M, g)$ is Ricci-flat and $\operatorname{ker} s_{g}^{\prime *}=\mathbb{R} \cdot 1$, or the scalar curvature is a strictly positive constant and $s_{g} /(n-1)$ is an eigenvalue of the Laplacian. J. Lafontaine ([9]) has shown that if a 3-dimensional compact manifold $(M, g)$ satisfies $\operatorname{dim} \operatorname{ker} s_{g}^{*} \geq 2$, then $M$ is isometric to a standard product $S^{1} \times S^{2}, S^{1} \times \mathbb{R} \mathbb{P}^{2}$ or to the standard 3-sphere. By combining this result with the fact that a CPE solution cannot be realized on a (warped) product metric space ([3]), it is easy to see that a three-dimensional solution metric of CPE with $\operatorname{dim} \operatorname{ker} s_{g}^{\prime *} \geq 2$ is Einstein. On the other hand, the authors ([6]) have shown that if a 3-dimensional solution metric $g$ of the CPE satisfies $\operatorname{ker} s_{g}^{\prime *} \neq 0$ and $H_{2}(M, \mathbb{Z})=0$, then $(M, g)$ is isometric to a standard sphere.

In this paper, we study some properties for functions in $\operatorname{ker} s_{g}^{*}$ and their relations to the geometric structure of underlying manifold. The zero set of a function in $\operatorname{ker} s_{g}^{\prime *}$ has generically a special property. More precisely, if $\varphi \in \operatorname{ker} s_{g}^{*}$, then the zero set $\varphi^{-1}(0)$ is a totally geodesic hypersurface in the underlying manifold, and on the zero set $\varphi^{-1}(0)$, the Ricci tensor $r_{g}(X, \nabla \varphi)=0$ for any tangent vector $X$ to $\varphi^{-1}(0)$. Using this property, we show that the norms of the Ricci tensor and the traceless Ricci tensor are constants on the zero set $\varphi^{-1}(0)$. From this fact together with the maximum principle, we can show that $z_{g}(\nu, v)=$ 0 on the set $\varphi^{-1}(0)$, where $v$ is a unit normal vector field on $\varphi^{-1}(0)$. Thus we have the following.

THEOREM 1.1. Let $(g, f)$ be a nontrivial solution of the critical point equation on a compact n-dimensional manifold $M$ with $n \geq 3$. If $\operatorname{ker} s_{g}^{*} \neq 0$, then $(M, g)$ is isometric to a standard sphere $S^{n}$ when $s_{g}>0$, and $(M, g)$ is Ricci-flat when $s_{g}=0$. In the latter case, $f$ should be constant.

As an application, we prove that if the CPE has two distinct solutions satisfying (1), then $(M, g)$ is Einstein. Note that there exists two distinct solutions satisfying the critical point equation if and only if $\operatorname{ker} s_{g}^{\prime *} \neq 0$. Finally, we would like to mention that our result works for arbitrary dimension $n \geq 3$ rather than $n=3$, generalizing previous results. 
2. Structural properties of functions in $\operatorname{ker} s_{g}^{*}$. In this section we derive several important identities involving the Ricci tensor $r_{g}$ or the traceless Ricci tensor $z_{g}$ and their derivatives when $\operatorname{ker} s_{g}^{\prime *} \neq 0$.

First, we fix our convention as follows. Throughout the paper, the Riemann curvature tensor is given by

$$
R(X, Y) Z=D_{Y} D_{X} Z-D_{X} D_{Y} Z+D_{[X, Y]} Z
$$

and the Ricci curvature is

$$
r(X, Y)=\sum_{i=1}^{n}\left\langle R\left(X, E_{i}\right) Y, E_{i}\right\rangle
$$

as in [1]. However, the Laplacian of a function $f$ is defined to be $\Delta f=-\delta d f$; there is a difference in sign between [1] and ours.

We will focus our attention on $\delta d^{D} r_{g}$ and $\delta d^{D} z_{g}$ and the structure of zero-level sets of functions in $\operatorname{ker} s_{g}^{\prime *}$. The differential operator $d^{D}$ of $C^{\infty}\left(S^{2} M\right)$ into $\Lambda^{2} M \otimes T^{*} M$ is defined as

$$
d^{D} \eta(X, Y, Z)=\left(D_{X} \eta\right)(Y, Z)-\left(D_{Y} \eta\right)(X, Z)
$$

for sections of symmetric 2-tensors $\eta \in C^{\infty}\left(S^{2} M\right)$ and the operator $\delta$ is its formal adjoint. We also define an interior product $\tilde{i}$ to the final factor by

$$
\tilde{i}_{\xi} \omega(X, Y, Z)=\omega(X, Y, Z, \xi)
$$

for a 4-tensor $\omega$ and a vector field $\xi$.

Let $\varphi$ be a nontrivial function in $\operatorname{ker} s_{g}^{\prime *}$ so that $\varphi$ satisfies the following equation:

$$
\varphi z_{g}=D_{g} d \varphi+\frac{s_{g} \varphi}{n(n-1)} g .
$$

First of all, note that there are no critical points of $\varphi$ in the set $\Gamma:=\varphi^{-1}(0)$ ([4]). Moreover on the set $\Gamma:=\varphi^{-1}(0)$, we have $D d \varphi=0$ and so any connected component of $\Gamma:=\varphi^{-1}(0)$ is totally geodesic. From now on, we denote $s_{g}$ by $s, r_{g}$ by $r$, and $z_{g}$ by $z$ unless confused. Then, from (3) we have the following structure equation.

LEMMA 2.1. If $\varphi \in \operatorname{ker} s_{g}^{*}$, we have

$$
\varphi d^{D} r=\tilde{i}_{\nabla \varphi} \mathcal{W}-\frac{n-1}{n-2} d \varphi \wedge z-\frac{1}{n-2} i_{\nabla \varphi} z \wedge g .
$$

Here $\mathcal{W}$ is the Weyl tensor of the metric $g$ and $i_{X}$ is the (usual) interior product with respect to a vector field $X$.

Proof. Applying $d^{D}$ to both sides of (3) and using the Ricci identity

$$
d^{D} \operatorname{Dd} \varphi(X, Y, Z)=R(X, Y, Z, \nabla \varphi)
$$

for any vector fields $X, Y, Z$ on $M$, we obtain

$$
\left(d \varphi \wedge z+\varphi d^{D} z\right)(X, Y, Z)=R(X, Y, Z, \nabla \varphi)+\frac{s}{n(n-1)}(d \varphi \wedge g)(X, Y, Z) .
$$


Here $d \varphi \wedge \eta$ is defined as

$$
(d \varphi \wedge \eta)(X, Y, Z)=d \varphi(X) \eta(Y, Z)-d \varphi(Y) \eta(X, Z)
$$

for $\eta \in C^{\infty}\left(S^{2} M\right)$. From the curvature decomposition (c.f. [1, 1.116, p. 48])

$$
\begin{aligned}
R(X, Y, Z, W)= & \mathcal{W}(X, Y, Z, W)+\frac{1}{n-2}(g(X, Z) r(Y, W)+g(Y, W) r(X, Z) \\
& -g(Y, Z) r(X, W)-g(X, W) r(Y, Z)) \\
& -\frac{s}{(n-1)(n-2)}(g(X, Z) g(Y, W)-g(Y, Z) g(X, W)),
\end{aligned}
$$

we obtain

$$
\tilde{i}_{\nabla \varphi} R=\tilde{i}_{\nabla \varphi} \mathcal{W}-\frac{1}{n-2} i_{\nabla \varphi} r \wedge g-\frac{1}{n-2} d \varphi \wedge r+\frac{s}{(n-1)(n-2)} d \varphi \wedge g .
$$

By combining these results together with $z=r-(s / n) g$, we obtain (4).

As an application of Lemma 2.1, we have the following property: Let $X$ be a vector field which is tangent to $\Gamma$ so that $\langle X, \nabla \varphi\rangle=0$. Substituting the triple $(X, \nabla \varphi, \nabla \varphi)$ into (4), we obtain

$$
0=\tilde{i}_{\nabla \varphi} \mathcal{W}(X, \nabla \varphi, \nabla \varphi)+z(\nabla \varphi, X)|\nabla \varphi|^{2}
$$

on the set $\Gamma$. Consequently, we have, for a vector $X$ tangent to $\Gamma$,

$$
z(X, \nabla \varphi)=0
$$

on the set $\Gamma=\varphi^{-1}(0)$.

COROLLARY 2.2. Let $\varphi \in \operatorname{ker} s_{g}^{*} \backslash\{0\}$. Then $\varphi^{-1}(0)$ is a collection of totally geodesic hypersurfaces in $M$, and for a vector $X$ which is tangent to $\varphi^{-1}(0)$,

$$
z(X, \nabla \varphi)=0
$$

on the $\operatorname{set} \varphi^{-1}(0)$.

For any two symmetric 2-tensors $h, k$, the composition $h \circ k$ is defined by

$$
h \circ k(X, Y)=\langle h(X), k(Y)\rangle=\sum_{i=1}^{n} h\left(X, E_{i}\right) k\left(Y, E_{j}\right)
$$

for any vectors $X, Y$ and an orthonormal frame $\left\{E_{i}\right\}$.

LEMMA 2.3. We have

$$
\varphi \delta d^{D} r+T=3 D_{\nabla \varphi} z-\frac{s \varphi}{n-1} z-\frac{n}{n-2} \varphi z \circ z+\frac{1}{n-2} \varphi|z|^{2} g,
$$

where $T$ is a symmetric 2-tensor defined by

$$
T(X, Y)=D_{X} z(\nabla \varphi, Y)+D_{Y} z(\nabla \varphi, X)-\varphi(\stackrel{\mathcal{W}}{z})(X, Y)
$$

with

$$
(\mathcal{W} z)(X, Y)=\sum_{i=1}^{n} z\left(\mathcal{W}\left(X, E_{i}\right) Y, E_{i}\right)
$$


for an orthonormal frame $\left\{E_{i}\right\}$.

PROOF. Let $\left\{E_{i}\right\}$ be an orthonormal frame. Taking the divergence of (4), the left-hand side becomes

$$
\begin{aligned}
\delta\left(\varphi d^{D} r\right) & =\varphi \delta d^{D} r-i_{\nabla \varphi} d^{D} r \\
& =\varphi \delta d^{D} r-i_{\nabla \varphi} d^{D} z
\end{aligned}
$$

since $s_{g}$ is constant. Next, identifying the sections of $T^{*} M \otimes \Lambda^{2} M$ with sections of $\Lambda^{2} M \otimes$ $T^{*} M$, we have $([1,16.3$, p. 435])

$$
\delta \mathcal{W}(X, Y, \nabla \varphi)=-\frac{n-3}{n-2} d^{D} r(Y, \nabla \varphi, X)
$$

for any tangent vectors $X, Y$ (cf. [1]). Here, $d^{D}(s g)=0$ since $s$ is constant. Thus, from the fact $\sum_{i=1}^{n} \mathcal{W}\left(E_{i}, X, Y, E_{i}\right)=0$ and (3), we have

$$
\begin{aligned}
\delta \tilde{i}_{\nabla \varphi} \mathcal{W}(X, Y) & =-\sum_{i} D_{E_{i}} \tilde{i}_{\nabla \varphi} \mathcal{W}\left(E_{i}, X, Y\right) \\
& =-\frac{n-3}{n-2} d^{D} r(Y, \nabla \varphi, X)-\sum_{i} \mathcal{W}\left(E_{i}, X, Y, D_{E_{i}} \nabla \varphi\right) \\
& =-\frac{n-3}{n-2} d^{D} r(Y, \nabla \varphi, X)+\varphi\left(\stackrel{\mathcal{W}}{z}_{z}\right)(X, Y) \\
& =-\frac{n-3}{n-2} D_{Y} r(\nabla \varphi, X)+\frac{n-3}{n-2} D_{\nabla \varphi} r(Y, X)+\varphi\left(\mathcal{W}_{z}\right)(X, Y) .
\end{aligned}
$$

Also we can compute

$$
\begin{aligned}
\delta(d \varphi \wedge z) & =\frac{s}{n-1} \varphi z-D_{\nabla \varphi} z+D d \varphi \circ z \\
& =\frac{s}{n} \varphi z-D_{\nabla \varphi} z+\varphi z \circ z
\end{aligned}
$$

and

$$
\begin{aligned}
\delta\left(i_{\nabla \varphi} z \wedge g\right)(X, Y)= & -\langle D d \varphi, z\rangle g(X, Y)+D d \varphi \circ z(X, Y)+D_{Y} z(\nabla \varphi, X) \\
= & -\varphi|z|^{2} g(X, Y)+\varphi z \circ z(X, Y) \\
& +D_{Y} z(\nabla \varphi, X)-\frac{s}{n(n-1)} \varphi z(X, Y) .
\end{aligned}
$$

Substituting these into the divergence of (4), we obtain (6).

As a direct consequence of Lemma 2.3, we have

COROLlaRy 2.4. On $\Gamma=\varphi^{-1}(0)$, for any vector $X$ and $Y$ (not necessarily tangent to $\Gamma$ ),

$$
3 D_{\nabla \varphi} z(X, Y)=D_{Y} z(\nabla \varphi, X)+D_{X} z(\nabla \varphi, Y) .
$$

Since the set of all regular values is open, the vector field $v:=\nabla \varphi /|\nabla \varphi|$ is well-defined near a tubular neighborhood of $\Gamma=\varphi^{-1}(0)$ and is a unit normal vector field on $\Gamma$. Now we will compute $\delta d^{D} r(v, v)$ in two different ways near a tubular neighborhood of $\Gamma$. We will 
obtain one from function properties in $\operatorname{ker} s_{g}^{*}$ together with Lemma 2.3. The second identity follows from a general fact described in [1] and (5). On the other hand, we will obtain another identity for $\delta d^{D} r(v, v)$ by using the CPE (1) and some function properties in $\operatorname{ker} s_{g}^{\prime *}$.

Let $\rho=z(v, v)$. First, substituting $(v, v)$ into $(6)$ and taking $v$-directional derivative, we have

$$
\begin{aligned}
|\nabla \varphi| \delta d^{D} r(v, v)+\varphi v\left(\delta d^{D} z(v, v)\right) \\
=3 v\left(D_{\nabla \varphi} z(v, v)\right)-2 v\left(D_{\nu} z(\nabla \varphi, v)\right)+|\nabla \varphi|\left(\mathcal{W}_{z}\right)(v, v) \\
\quad-\frac{s|\nabla \varphi|}{n-1} \rho-\frac{n|\nabla \varphi|}{n-2} \rho^{2}+\frac{|\nabla \varphi|}{n-2}|z|^{2}-\varphi \cdot v(Q(v, v))
\end{aligned}
$$

on a tubular neighborhood of $\Gamma$. Here

$$
Q=\frac{s}{n-1} z+\frac{n}{n-2} z \circ z-\frac{1}{n-2}|z|^{2} g-\mathcal{W} z
$$

Note that on the set $\Gamma=\varphi^{-1}(0), v(|\nabla \varphi|)=\left\langle D_{\nu} \nabla \varphi, v\right\rangle=0$ and $D_{\nu} v=0$ by (5). It is clear that

$$
D_{\nabla \varphi} z(\nu, v)=D_{\nu} z(\nabla \varphi, v)
$$

on the tubular neighborhood of $\Gamma$. Thus we obtain

$$
\begin{aligned}
\delta d^{D} r(v, v)= & \frac{1}{|\nabla \varphi|} v\left(D_{\nabla \varphi} z(v, v)\right)-\frac{s}{n-1} \rho \\
& -\frac{n}{n-2} \rho^{2}+\frac{1}{n-2}|z|^{2}+\left(\mathcal{W}_{z}\right)(v, v)
\end{aligned}
$$

on $\Gamma$. Moreover, since $\left\langle D_{\nu} D_{\nabla \varphi} v, v\right\rangle=v\left\langle D_{\nabla \varphi} v, v\right\rangle=0$ on $\Gamma=\varphi^{-1}(0)$, we have

$$
\begin{aligned}
v\left(D_{\nabla \varphi} z(\nu, v)\right) & =v\left(\nabla \varphi(z(\nu, v))-2 v\left(z\left(D_{\nabla \varphi} v, v\right)\right)\right. \\
& =v\langle\nabla \varphi, \nabla \rho\rangle-2 z\left(D_{\nu} D_{\nabla \varphi} v, v\right)=\left\langle D_{v} \nabla \rho, \nabla \varphi\right\rangle
\end{aligned}
$$

since $D_{\nabla \varphi} v=0$. Also, by (4) and (5), we have, on $\Gamma$

$$
\begin{aligned}
\mathcal{W}_{z}(v, v) & =\frac{1}{|\nabla \varphi|} \sum_{i, j} \mathcal{W}\left(E_{i}, v, E_{j}, \nabla \varphi\right) z\left(E_{i}, E_{j}\right) \\
& =\frac{1}{|\nabla \varphi|}\left(\frac{n}{n-2} \sum_{i}|\nabla \varphi| z\left(\nu, E_{i}\right)^{2}-\frac{n-1}{n-2}|\nabla \varphi||z|^{2}\right) \\
& =\frac{n}{n-2} \rho^{2}-\frac{n-1}{n-2}|z|^{2} .
\end{aligned}
$$

Thus we obtain the first identity for $\delta d^{D} r(v, v)$ by substituting these into (7).

LEMMA 2.5. On $\Gamma=\varphi^{-1}(0)$, we have

$$
\delta d^{D} r(v, v)=\left\langle D_{v} \nabla \rho, v\right\rangle-\frac{s}{n-1} \rho-|z|^{2} .
$$

For the second identity for $\delta d^{D} r(v, v)$, we first observe the following lemma. 
LEMMA 2.6 ([1, 4.71, p. 134]). We have

$$
\delta d^{D} r=D^{*} D r+\frac{1}{2} D d s+r \circ r-\stackrel{\circ}{R} r .
$$

Note that the value of $\delta d^{D} r$ in [1] should be divided by 2. Since the scalar curvature $s$ is constant, and

we have, from (9)

$$
r \circ r=z \circ z+\frac{2 s}{n} z+\frac{s^{2}}{n^{2}} g
$$

$$
\delta d^{D} r=D^{*} D z+z \circ z+\frac{2 s}{n} z+\frac{s^{2}}{n^{2}} g-\stackrel{\circ}{R} r .
$$

Thus, by the identity relation given by

$$
\stackrel{\circ}{R} r=\mathcal{W} z+\frac{1}{n-2}|z|^{2} g+\frac{(n-2) s}{n(n-1)} z-\frac{2}{n-2} z \circ z+\frac{s^{2}}{n^{2}} g,
$$

we have

$$
\delta d^{D} r=D^{*} D z+\frac{n}{n-2} z \circ z+\frac{s}{n-1} z-\frac{1}{n-2}|z|^{2} g-\mathcal{W} z .
$$

Therefore, by substituting (8) into (10), we obtain the following.

LEMMA 2.7. On $\Gamma$ we have

$$
\delta d^{D} r(v, v)=-\Delta \rho+\frac{s}{n-1} \rho+|z|^{2} .
$$

REMARK 2.8. Comparing Lemma 2.5 with Lemma 2.7, we have

$$
\frac{1}{2} \Delta^{\prime} \rho=\left\langle D_{v} \nabla \rho, v\right\rangle+\frac{s}{n-1} \rho+|z|^{2}
$$

on $\Gamma$.

3. The CPE and derivative of $z_{g}(v, v)$. In this section, we will derive another identity for $\delta d^{D} r(v, v)$ and investigate its relation to the critical point equation. Let $f$ be a solution of the critical point equation (1). First, we start with the following observation from the critical point equation

$$
(1+f) z(v, v)=-\Delta^{\prime} f-\frac{s}{n} f
$$

on $\Gamma$. In fact, it follows from (1) that $\Delta f=-\frac{s}{n-1} f$ and so

$$
(1+f) z_{g}=D d f+\frac{s_{g} f}{n(n-1)} g .
$$

Since $\Gamma$ is totally geodesic,

which implies (11).

$$
\begin{aligned}
-\frac{s}{n-1} f=\Delta f & =\Delta^{\prime} f+\operatorname{Ddf}(\nu, v) \\
& =\Delta^{\prime} f+(1+f) \rho-\frac{s}{n(n-1)} f
\end{aligned}
$$


LEMma 3.1 ([5]). Assume that $f$ is a solution to the CPE and $\varphi \in \operatorname{ker} s_{g}^{*} \backslash\{0\}$. Then $|\nabla \varphi|^{2}$ and $c:=\langle\nabla f, \nabla \varphi\rangle /|\nabla \varphi|^{2}$ are constants on each connected component of $\Gamma=\varphi^{-1}(0)$.

LEMMA 3.2. Assume that $f$ is constant on a connected open subset $\mathcal{U}$ of $\Gamma$. Then $\rho=z(v, v)$ and $|z|^{2}$ are constants on $\mathcal{U}$.

ProOF. Suppose that $f$ is constant on an open subset $\mathcal{U}$ of $\Gamma$. Since $\Delta^{\prime} f=0$ on $\mathcal{U}$, it follows from (11) that $1+f$ cannot be zero and so

$$
\rho=z(v, v)=-\frac{s}{n} \frac{f}{1+f} .
$$

In other words, $\rho$ is constant on $\mathcal{U}$ if $f$ is constant on $\mathcal{U}$.

Next, we claim that $|z|^{2}$ is constant on $\mathcal{U}$. By the Bochner formula and (1),

$$
\begin{aligned}
(1+f)^{2}|z|^{2} & =|D d f|^{2}-\frac{s^{2} f^{2}}{n(n-1)^{2}} \\
& =\frac{1}{2} \Delta|\nabla f|^{2}-\rho|\nabla f|^{2}+\frac{s}{n(n-1)}|\nabla f|^{2}-\frac{s^{2} f^{2}}{n(n-1)^{2}} .
\end{aligned}
$$

Since

$$
|\nabla f|^{2}=c^{2}|\nabla \varphi|^{2}=\langle\nabla f, v\rangle^{2},
$$

it follows from Lemma 3.1 that $|\nabla f|^{2}$ is constant on $\mathcal{U}$.

Using the fact that

$$
(1+f) \rho=-\frac{s}{n} f=\operatorname{Ddf}(v, v)+\frac{s f}{n(n-1)}
$$

on $\mathcal{U}$ and

$$
|\nabla f| v(|\nabla f|)=\frac{1}{2} v\left(|\nabla f|^{2}\right)=\left\langle D_{v} \nabla f, \nabla f\right\rangle,
$$

we have

$$
\begin{aligned}
\frac{1}{2} \Delta|\nabla f|^{2} & =\frac{1}{2} \Delta^{\prime}|\nabla f|^{2}+\frac{1}{2}\left\langle D_{v} \nabla|\nabla f|^{2}, v\right\rangle \\
& =v\left\langle D_{v} \nabla f, \nabla f\right\rangle=v\left[v(f)\left((1+f) \rho-\frac{s f}{n(n-1)}\right)\right] \\
& =\frac{s^{2} f^{2}}{(n-1)^{2}}+|\nabla f|^{2} \rho+(1+f) v(f) v(\rho)-\frac{s}{n(n-1)}|\nabla f|^{2},
\end{aligned}
$$

where we used the fact that $(v(f))^{2}=|\nabla f|^{2}$ and

$$
v(v(f))=\left\langle D_{v} \nabla f, v\right\rangle=\operatorname{Ddf}(v, v)=-\frac{s f}{n-1}
$$

on $\mathcal{U}$ in the last equality, since $f$ is constant and $D d \varphi=0$ on $\mathcal{U}$. Thus, we obtain

$$
(1+f)^{2}|z|^{2}=\frac{s^{2} f^{2}}{n(n-1)}+(1+f) v(f) v(\rho)
$$


on $\mathcal{U}$. However, $v(\rho)=0$ on $\Gamma$ since $3 D_{\nabla \varphi} z(v, v)=2 D_{v} z(\nabla \varphi, v)$ on $\Gamma$ by Corollary 2.4. Therefore, on $\mathcal{U}$ we obtain

$$
|z|^{2}=\frac{s^{2} f^{2}}{n(n-1)(1+f)^{2}}=\frac{n}{n-1} \rho^{2},
$$

which implies that $|z|^{2}$ is constant on $\mathcal{U}$ since $f$ and $\rho$ are constants.

REMARK 3.3. Let $\xi:=\nabla f-c \nabla \varphi$ which is the tangential component of $\nabla f$ to $\Gamma=\varphi^{-1}(0)$, and consider an open subset $\mathcal{O}$ of $\Gamma$ defined by $\mathcal{O}=\{x \in \Gamma: \xi(x) \neq 0\}$. By definitions of $\mathcal{O}$ and $c$, it is clear that $f$ is not constant on $\mathcal{O}$. Recall that $c$ is constant on $\Gamma$, and so if $\nabla f$ is orthogonal to $\nabla \varphi$ at a point in $\Gamma$, then $\nabla f$ is orthogonal to $\nabla \varphi$ on all of $\Gamma$. From this fact, we may assume, without loss of generality, that $c \neq 0$ on a connected component of $\Gamma$. In fact, if $c=0$ on $\Gamma$, then a new function $\tilde{f}=f+k \varphi$ with a non-zero constant $k$ is again a solution of (1) and satisfies

$$
\tilde{c}=\frac{\langle\nabla \tilde{f}, \nabla \varphi\rangle}{|\nabla \varphi|^{2}}=k \neq 0 .
$$

The following property plays a crucial role in investigating geometric structure of $\Gamma:=$ $\varphi^{-1}(0)$ and properties for $\rho=z(v, v)$.

Proposition 3.4. On $\Gamma$, for $\xi:=\nabla f-c \nabla \varphi$ with $c:=\langle\nabla f, \nabla \varphi\rangle /|\nabla \varphi|^{2} \neq 0$, we have

$$
\xi(\rho)=-2 \rho\left((1+f) \rho-\frac{s f}{n(n-1)}\right) .
$$

PROOF. It follows from Corollary 2.4 that

$$
D_{\nabla \varphi} z(\nabla \varphi, \nabla \varphi)=0 .
$$

Since $|\nabla \varphi|$ is constant and $D d \varphi=0$ on $\Gamma$, it is easy to see from (16) that

$$
\xi(\rho)=\xi(z(\nu, \nu))=\frac{1}{|\nabla \varphi|^{2}} D_{\nabla f} z(\nabla \varphi, \nabla \varphi) .
$$

By Corollary 2.4 again, we have

$$
\begin{aligned}
2 D_{\nabla \varphi} z(\nabla f, \nabla \varphi) & =2 D_{\nabla \varphi} z(\xi, \nabla \varphi) \\
& =D_{\xi} z(\nabla \varphi, \nabla \varphi)=D_{\nabla f} z(\nabla \varphi, \nabla \varphi) .
\end{aligned}
$$

Since $\xi$ is tangent to $\Gamma$ and $D d \varphi=0$, we have $z(\nabla \varphi, \xi)=0$ by Coroallary 2.2. Thus

$$
D_{\xi} z(\nabla \varphi, \xi)=0
$$

and

$$
3 D_{\nabla \varphi} z(\xi, \xi)=2 D_{\xi} z(\nabla \varphi, \xi)=0
$$

Moreover, since

$$
z\left(D_{\nabla \varphi} \nabla f, \xi\right)=z\left(D_{\nabla \varphi} \xi, \xi\right)=\left\langle D_{\nabla \varphi} \nabla f, v\right\rangle z(\nu, \xi)=0,
$$


we obtain

$$
0=D_{\nabla \varphi} z(\xi, \xi)=\nabla \varphi(z(\xi, \xi))-2 z\left(D_{\nabla \varphi} \xi, \xi\right)=\nabla \varphi(z(\xi, \xi)) .
$$

By (16) and (18),

$$
\begin{aligned}
0=D_{\nabla \varphi} z(\xi, \xi) & =D_{\nabla \varphi} z(\nabla f, \nabla f)-2 c D_{\nabla \varphi} z(\nabla f, \nabla \varphi) \\
& =D_{\nabla \varphi} z(\nabla f, \nabla f)-c D_{\nabla f} z(\nabla \varphi, \nabla \varphi)
\end{aligned}
$$

In other words,

$$
D_{\nabla \varphi} z(\nabla f, \nabla f)=c D_{\nabla f} z(\nabla \varphi, \nabla \varphi) .
$$

Therefore, since $z(\xi, \xi)=z(\nabla f, \nabla f)-c^{2} z(\nabla \varphi, \nabla \varphi)$, it follows from (16) and (20),

$$
\begin{aligned}
0=\nabla \varphi(z(\xi, \xi)) & =\nabla \varphi(z(\nabla f, \nabla f)) \\
& =c D_{\nabla f} z(\nabla \varphi, \nabla \varphi)+2 z\left(D_{\nabla \varphi} \nabla f, \nabla f\right) .
\end{aligned}
$$

Finally, it follows from (1) that

$$
(1+f) z=D d f+\frac{s f}{n(n-1)} g .
$$

By Corollary 2.2 and (22), $D d f(\xi, \nabla \varphi)=0$ and $z\left(D_{\nabla \varphi} \nabla f, \xi\right)=0$ on $\Gamma$ and so

$$
\begin{aligned}
z\left(D_{\nabla \varphi} \nabla f, \nabla f\right) & =z\left(D_{\nabla \varphi} \nabla f, \xi\right)+c z\left(D_{\nabla \varphi} \nabla f, \nabla \varphi\right) \\
& =c\left\langle D_{\nabla \varphi} \nabla f, \nabla \varphi\right\rangle z(\nu, v) \\
& =c|\nabla \varphi|^{2}\left((1+f) z(\nu, v)-\frac{s f}{n(n-1)}\right) z(\nu, v) .
\end{aligned}
$$

Substituting this into (21), from the assumption $c \neq 0$, we may conclude that

$$
\frac{1}{2} D_{\nabla f} z(\nabla \varphi, \nabla \varphi)+\left((1+f) z(v, v)-\frac{s f}{n(n-1)}\right) z(\nabla \varphi, \nabla \varphi)=0,
$$

which proves our proposition by (17).

We remark that $\xi$ may be trivial in Proposition 3.4. As an immediate consequence, we obtain the following interesting result:

COROLlary 3.5. Suppose that $f$ is constant on an open subset $\mathcal{U}$ of $\Gamma$. Then we have $f=\rho=|z|^{2}=0$ on $\mathcal{U}$.

ProOF. Since $f$ is constant on $\mathcal{U}$, by (13)

$$
\rho=-\frac{s}{n} \cdot \frac{f}{1+f}
$$

It is clear that $\xi=0$ on $\mathcal{U}$, since $\nabla f$ is parallel to $\nabla \varphi$. Thus, by Proposition 3.4

$$
0=-2\left(\frac{s f}{n-1}\right) \cdot \frac{s}{n} \cdot \frac{f}{1+f}=-\frac{2 s^{2} f^{2}}{n(n-1)(1+f)},
$$

which implies that $f=\rho=0$. By (14), $|z|^{2}$ is also constant and equal to zero on $\mathcal{U}$. 
Now assume that $f$ is a solution to (1). A similar computation as Lemma 2.1 shows that

$$
(1+f) d^{D} r=\tilde{i}_{\nabla f} \mathcal{W}-\frac{n-1}{n-2} d f \wedge z-\frac{1}{n-2} i_{\nabla f} z \wedge g .
$$

Thus, similarly as in Lemma 2.3, we obtain

$$
\begin{aligned}
(1+f) \delta d^{D} r+S= & 3 D_{\nabla f} z-\frac{s f}{n-1} z \\
& -\frac{n}{n-2}(1+f) z \circ z+\frac{1}{n-2}(1+f)|z|^{2} g,
\end{aligned}
$$

where

$$
S(X, Y)=D_{X} z(\nabla f, Y)+D_{Y} z(\nabla f, X)-(1+f)\left(\mathcal{W}_{z}\right)(X, Y)
$$

By (18),

$$
2 D_{v} z(\nabla f, v)=D_{\nabla f} z(v, v)
$$

Also, it is easy to see that

$$
D_{\xi} z(v, v)=D_{\nabla f} z(v, v)=\nabla f(\rho)-2 z\left(D_{\nabla f} v, v\right)=\nabla f(\rho) .
$$

Therefore, by (8) and (24) we have on $\Gamma$

$$
2 \xi(\rho)=(1+f) \delta d^{D} r(v, v)+\frac{s f}{n-1} \rho+(1+f)|z|^{2} .
$$

It follows from Proposition 3.4 and (25) that the following third identity for $\delta d^{D} r(v, v)$ holds.

LEMMA 3.6. On $\Gamma$,

$$
\delta d^{D} r(v, v)=-4 \rho^{2}-\frac{(n-4)}{n(n-1)} \cdot \frac{s f \rho}{1+f}-|z|^{2} .
$$

Consider a connected component $\Sigma$ of $\Gamma$ and we may assume that $c \neq 0$ by Remark 3.3. Since $\Delta \rho=\Delta^{\prime} \rho+\left\langle D_{\nu} d \rho, v\right\rangle$, combining Lemma 2.5, Lemma 2.7, and Lemma 3.6 gives the following result:

THEOREM 3.7. On a connected component $\Sigma$ of $\Gamma$ with $c \neq 0$, we have

$$
\frac{1}{2} \Delta^{\prime} \rho=|z|^{2}+4 \rho^{2}+\frac{(n-4)}{n(n-1)} \cdot \frac{s f \rho}{1+f} .
$$

4. Structure of fibers and non-positivity of $z_{g}(\nu, v)$. Let $\varphi \in \operatorname{ker} s_{g}^{*} \backslash\{0\}$. Recall that $\varphi^{-1}(0)$ is a collections of connected totally geodesic hypersurfaces in $M$. In this section, we will prove that $\rho=z(\nu, \nu)$ is non-positive on each connected component of $\varphi^{-1}(0)$ by using the maximun principle, and conclude that $\rho$, in fact, vanishes on each component. Then using this fact together with the divergence theorem, we will prove our main theorem.

Let $\Sigma$ be a connected component of $\Gamma=\varphi^{-1}(0)$. We decompose $\Sigma$ into three disjoint subsets as follows: For a unit normal vector field $v=\nabla \varphi /|\nabla \varphi|$ on $\Sigma$,

$$
\begin{aligned}
& \Sigma^{A}=\{x \in \Sigma: \nabla f \text { is parallel to } v \text { with } \nabla f \neq 0 \text { at } x\}, \\
& \Sigma^{B}=\{x \in \Sigma: \nabla f \text { is not parallel to } v \text { with } \nabla f \neq 0 \text { at } x\}, \\
& \Sigma^{C}=\{x \in \Sigma: \nabla f(x)=0\} .
\end{aligned}
$$


Recalling $\xi:=\nabla f-c \nabla \varphi$ with $c=\langle\nabla f, \nabla \varphi\rangle /|\nabla \varphi|^{2}$, the tangential component of $\nabla f$ to $\Sigma$, it is clear that $\Sigma^{B}=\{x \in M: \xi(x) \neq 0\}$. We have also seen in the previous section that for the unit normal vector field $v$ on $\Gamma,\langle\nabla f, v\rangle$ is constant on $\Gamma$ (cf. [5]). Note that $\langle\nabla f, v\rangle \neq 0$ on $\Sigma^{A}$ since $\nabla \varphi \neq 0$ on $\Sigma$, and obviously $\langle\nabla f, v\rangle=0$ on $\Sigma^{C}$. Thus if there exists a point $x \in \Sigma$ such that $\langle\nabla f, v\rangle(x)=0$, then $\langle\nabla f, v\rangle=0$ on the whole $\Sigma$, and so $\Sigma=\Sigma^{B} \cup \Sigma^{C}$. Consequently, we have either $\Sigma=\Sigma^{A} \cup \Sigma^{B}$ or $\Sigma=\Sigma^{B} \cup \Sigma^{C}$. Furthermore, for the case in which $\Sigma=\Sigma^{B} \cup \Sigma^{C}, \nabla f$ is tangent to $\Sigma^{B}$ because $\Sigma^{A}=\emptyset,\langle\nabla f, v\rangle$ is constant on $\Sigma$, and is equal to zero on $\Sigma^{C}$. Thus considering a new function $\bar{f}=f+c \varphi$ which is also a solution to (1) and satisfies that $\nabla \bar{f} \neq 0$, the latter case is reduced to the first case. In fact, we gave in this case, $\Sigma^{B}=\{x \in \Sigma: \nabla \bar{f}$ is not parallel to $v$ with $\nabla \bar{f} \neq 0$ at $x\}$ and $\Sigma^{C}=\{x \in \Sigma: \nabla \bar{f}$ is parallel to $v$ with $\nabla \bar{f} \neq 0$ at $x\}$. Therefore, we may assume that $\Sigma=\Sigma^{A} \cup \Sigma^{B}$.

To prove Theorem 1.1, we shall first show that $\rho=0$ on $\Sigma$ (Theorem 4.2). To do this, we need the following lemma which shows that $\rho=z(v, v)$ is nonnegative on the set $\Sigma$.

LEMMA 4.1. The maximum of $\rho$ is zero on $\Sigma$.

Proof. By Remark 3.3, we may assume that $c \neq 0$ on $\Sigma$. Let $\rho\left(x_{0}\right)=\max _{x \in \Sigma} \rho(x)$. First we consider the case in which the $(n-1)$-dimensional measure of $\Sigma^{A}$ is zero so that $\Sigma=\overline{\Sigma^{B}}$ and $x_{0} \in \overline{\Sigma^{B}}$. Since $\xi(\rho)\left(x_{0}\right)=\langle\xi, \nabla \rho\rangle\left(x_{0}\right)=0$, by Proposition 3.4, we have $\rho\left(x_{0}\right)=0$ or

$$
\rho\left(x_{0}\right)=\frac{s f\left(x_{0}\right)}{n(n-1)(1+f)\left(x_{0}\right)} .
$$

Recall that $(1+f)\left(x_{0}\right) \neq 0$ by (15). For either case, by Theorem 3.7

$$
\frac{1}{2} \Delta^{\prime} \rho\left(x_{0}\right)=|z|^{2}\left(x_{0}\right)+n \rho^{2}\left(x_{0}\right) \geq 0 .
$$

Since $\Delta^{\prime} \rho \leq 0$ at the maximum point $x_{0}$, we have

$$
\rho\left(x_{0}\right)=|z|^{2}\left(x_{0}\right)=0,
$$

which proves our lemma in the first case.

Now we consider the case when the $(n-1)$-dimenisonal measure of $\Sigma^{A}$ is not zero. In this case, since $|\nabla f|,|\nabla \varphi|$ and $c$ are all constants, and $\nabla f=c \nabla \varphi, f$ is constant on each connected component of $\Sigma^{A}$. Thus, by Corollary 3.5, $\rho=f=|z|^{2}=0$ on $\Gamma$.

The following shows that $\rho$ is identically zero on $\Gamma$.

THEOREM 4.2. On $\Gamma$ we have $\rho \equiv 0$.

ProOF. Let $\Sigma$ be a connected component of $\Gamma$. By Remark 3.3, we may assume that $c \neq 0$ on $\Sigma$. First we derive the following equation:

$$
\int_{\Sigma}(1+f)|z|^{2}=0 .
$$

By (11),

$$
\frac{s f \rho}{n}=-(1+f) \rho^{2}-\rho \Delta^{\prime} f
$$


Thus, by Theorem 3.7,

$$
\begin{aligned}
\frac{1}{2} \int_{\Sigma}(1+f) \Delta^{\prime} \rho & =\int_{\Sigma}(1+f)|z|^{2}+4(1+f) \rho^{2}+\frac{(n-4)}{(n-1)} \frac{s f \rho}{n} \\
& =\int_{\Sigma}(1+f)|z|^{2}+\frac{3 n}{n-1}(1+f) \rho^{2}-\frac{n-4}{n-1} \rho \Delta^{\prime} f .
\end{aligned}
$$

Note that

$$
\int_{\Sigma}(1+f) \Delta^{\prime} \rho=-\int_{\Sigma}\left\langle(\nabla f)^{T}, \nabla \rho\right\rangle_{\Sigma}=\int_{\Sigma} \rho \Delta^{\prime} f,
$$

where $\langle,\rangle_{\Sigma}$ is the induced metric of $g$ on $\Sigma$. Thus we obtain

$$
\frac{3(n-3)}{2(n-1)} \int_{\Sigma} \rho \Delta^{\prime} f=\int_{\Sigma}(1+f)|z|^{2}+\frac{3 n}{n-1}(1+f) \rho^{2} .
$$

We observe that the tangential part $(\nabla f)^{T}$ of $\nabla f$ to $\Sigma$ is equal to $\xi$. Thus, by Proposition 3.4 together with (28) we have

$$
\begin{aligned}
\int_{\Sigma} \rho \Delta^{\prime} f & =-\int_{\Sigma} \xi(\rho)=\int_{\Sigma} 2(1+f) \rho^{2}-\frac{2 s f \rho}{n(n-1)} \\
& =\int_{\Sigma} \frac{2 n}{n-1}(1+f) \rho^{2}+\frac{2}{n-1} \rho \Delta^{\prime} f
\end{aligned}
$$

which implies that

$$
(n-3) \int_{\Sigma} \rho \Delta^{\prime} f=2 n \int_{\Sigma}(1+f) \rho^{2} .
$$

Hence, by (29) and (30), we obtain

$$
\int_{\Sigma}(1+f)|z|^{2}=0 \text {. }
$$

In particular, we may conclude that if $1+f>0$ or $1+f<0$ on $\Sigma,|z|^{2}=\rho=0$ on $\Sigma$.

We claim that $\rho \equiv 0$ on all of $\Sigma$. Suppose, on the contrary, that $\rho$ is not identically zero on $\Sigma$. Recall that $\rho \leq 0$ on $\Sigma$ by Lemma 4.1. Let $x_{1}$ be a minimum point of $\rho$ in $\Sigma$ so that $\rho\left(x_{1}\right)<0$. It is clear that $x_{1}$ cannot lie in an open subset $\mathcal{U}$ of $\Sigma^{A}$ since $\rho=0$ on $\mathcal{U}$ by Corollary 3.5. Therefore $x_{1} \in \Sigma^{B}$ and so $\xi\left(x_{1}\right) \neq 0$. By Proposition 3.4, we have $\xi(\rho)\left(x_{1}\right)=0$ and so

$$
\rho\left(x_{1}\right)=\frac{s}{n(n-1)} \frac{f\left(x_{1}\right)}{(1+f)\left(x_{1}\right)}<0 .
$$

In particular, $-1<f\left(x_{1}\right)<0$.

Now consider the subset $\Omega=\{x \in \Sigma:(1+f)(x)>0\}$ of $\Sigma$ so that $x_{1} \in \Omega \neq \emptyset$. If $\Omega=\Sigma$, then $\rho=0$ on $\Sigma$ by argument above. Thus, we may assume that $\Omega \varsubsetneqq \Sigma$. Note that if $1+f=0$ at some point in $\Sigma$, then, by (26), we must have $\rho=0$ at the point. Thus, $\rho=0$ on $\partial \Omega$.

For a sufficiently small $\varepsilon$, letting

$$
\Omega_{\varepsilon}=\{x \in \Omega: 0<(1+f)(x)<\varepsilon\} \subset \Omega,
$$


we have

$$
\xi(\rho)=-2 \rho\left((1+f) \rho-\frac{s f}{n(n-1)}\right) \geq-2 \rho\left(\varepsilon \rho+\frac{s(1-\varepsilon)}{n(n-1)}\right) \geq 0 .
$$

However, since $\rho=0$ on $\partial \Omega$ and $\max _{\Sigma} \rho=0$ by Lemma 4.1, $\xi(\rho)$ cannot be positive on $\Omega_{\varepsilon}$. Therefore, $\xi(\rho) \equiv 0$ on $\Omega_{\varepsilon}$, implying that $\rho \equiv 0$ on $\Omega_{\varepsilon}$.

Now, for $-1 \leq a \leq f\left(x_{1}\right)$, let $L_{a}=f^{-1}(a) \cap \bar{\Omega}$, where $\bar{\Omega}=\Omega \cup \partial \Omega$. Clearly, by definition of $\Omega, L_{a}=\emptyset$ if $a<-1$. In terms of $L_{a}$, we have $x_{1} \in L_{f\left(x_{1}\right)}$ with $\rho\left(x_{1}\right)<0$, and any $y \in L_{-1}$ satisfies $\rho(y)=0$. Therefore, considering integral curves of $-\xi=-(\nabla f)^{T}$ from $x_{1}$ to $\partial \Omega$, there exists $x_{2}$ in some integral curve such that $x_{2} \in\left\{x \in L_{a}:-1<a<\right.$ $\left.f\left(x_{1}\right)\right\}$ and $\rho\left(x_{2}\right)<0$ and $\rho$ is decreasing at $x_{2}$ in the direction of $\xi=(\nabla f)^{T}$. In other words, $\xi(\rho)\left(x_{2}\right)<0$. Moreover, we may take $x_{2}$ such that $\rho\left(x_{2}\right)$ and so $\left(1+f\left(x_{2}\right)\right) \rho\left(x_{2}\right)$ are very close to zero. Thus, since $f\left(x_{2}\right)<f\left(x_{1}\right)<0$,

$$
\left(1+f\left(x_{2}\right)\right) \rho\left(x_{2}\right)-\frac{s}{n(n-1)} f\left(x_{2}\right)>0 .
$$

However, by Proposition 3.4, we have $\xi(\rho)>0$, which is a contradiction.

This contradiction shows that $\rho\left(x_{1}\right)=\min _{\Sigma} \rho=0$, and consequently $\rho \equiv 0$ on $\Sigma$. This completes the proof of Theorem 4.2.

THEOREM 4.3. Let $(g, f)$ be a nontrivial solution of the critical point equation on a compact n-dimensional manifold $M$ with $n \geq 3$. If $\operatorname{ker} s_{g}^{\prime *} \neq 0$, then $(M, g)$ is isometric to a standard sphere $S^{n}$ when $s_{g}>0$, and $(M, g)$ is Ricciflat when $s_{g}=0$. In the latter case, $f$ should be constant.

PROOF. Due to the result of Bouruignon mentioned in the introduction, it suffices to prove Theorem 4.3 only when $s_{g}>0$.

For a function $\varphi \in \operatorname{ker} s_{g}^{*} \backslash\{0\}$, let us denote

$$
M_{0, \varphi}=\{x \in M: \varphi(x)<0\} .
$$

Since $s_{g}$ is constant and so $\delta r_{g}=\delta z_{g}=-\frac{1}{2} d s_{g}=0$, we have, from (3)

$$
\delta\left(z_{g}(\nabla \varphi, \cdot)\right)=-\left\langle z_{g}, D d \varphi\right\rangle=-\varphi\left|z_{g}\right|^{2} .
$$

By divergence theorem and Theorem 4.2, we have

$$
\int_{M_{0, \varphi}} \varphi|z|^{2}=\int_{\varphi^{-1}(0)} \rho|\nabla \varphi|=0 .
$$

This implies that $z$ is identically zero on $M_{0, \varphi}$. This argument also can be used to prove that $z=0$ on $M_{\varphi}^{0}:=\{x \in M: \varphi(x)>0\}$. Thus, $g$ is Einstein and so $(M, g)$ is isometric to a standard sphere $S^{n}$.

\section{REFERENCES}

[ 1 ] A. L. BESSE, Einstein Manifolds, Ergeb. Math. Grenzgeb. (3) 10, Springer-Verlag, Berlin, 1987. 
[2] J. P. Bourguignon, Une stratification de léspace des structures riemanniennes, Compositio Math. 30 (1975), no. $1,1-41$.

[ 3 ] J. Chang, S. Hwang And G. Yun, Critical point metrics of the total scalar curvature, Bull. Korean Math. Soc. 49 (2012), no. 3, 655-667.

[ 4 ] A. E. Fischer AND J. E. MARSDEN, Manifolds of Riemannian metrics with prescribed scalar curvature, Bull. Am. Math. Soc. 80 (1974), 479-484.

[ 5 ] S. HwANG, The critical point equation on a three dimensional compact manifold, Proc. Amer. Math. Soc. 131 (2003), 3221-3230.

[6] S. Hwang, J. Chang AND G. Yun, Rigidity of the critical point equation, Math. Nachr. 283 (2010), no. 6, 846-853.

[ 7 ] N. KoIso, A decomposition of the space $\mathcal{M}$ of Riemannian metrics on a manifold, Osaka J. Math. 16 (1979), 423-429.

[ 8 ] J. Lafontaine, Sur la géométrie d'une généralisation de l'équation différentielle d'Obata, J. Math. Pures Appl. (9) 62 (1983), no. 1, 63-72.

[ 9 ] J. Lafontaine, A remark about static space times, J. Geom. Phys. 59 (2009), no. 1, 50-53.

[10] M. OBATA, Certain conditions for a Riemannian manifold to be isometric with a sphere, J. Math. Soc. Japan 14 (1962), no. 3, 333-340.

DePARTMENT OF MATHEMATics

MYONG JI UNIVERSITY

GYEONG-GI 449-728

Korea

E-mail address: gabjin@mju.ac.kr

Department of Mathematics

Chung-Ang UNIVERSiTY

SEOUL 156-756

KOREA

E-mail address: seungsu@cau.ac.kr
Department of Mathematics Education

DANKOOK UNIVERSITY

GYEONG-GI 448-701

KOREA

E-mail address: jchang@dankook.ac.kr 